\title{
LES SAINT-SIMONIENS \\ ET LA PHILOSOPHIE ALLEMANDE OU \\ LA PREMIÈRE ALLIANCE INTELLECTUELLE FRANCO-ALLEMANDE
}

En 1834 - dix ans avant la fondation par Marx et Ruge des Annales franco-allemandes -, un certain Moritz Veit, hégélien, juif et berlinois, publiait à Leipzig un essai au titre programmatique : Saint-Simon et le saint-simonisme. Alliance générale des peuples et paix éternelle ${ }^{1}$. Le soupçon d'une telle "alliance ", fondée sur et par les philosophies les plus avancées de France et d'Allemagne, avait commencé à se répandre dès $1831-1832^{2}$. Mais il faut une décennie pour qu'un exsaint-simonien en confirme la réalité. Témoignage tardif, qu'il y a lieu, donc, de critiquer. Mais Pierre Leroux n'est pas le premier venu, et l'on ne saurait refuser tout crédit à la révélation qu'il livre en 1842 : "Il faudra bien qu'un jour, on sache que la métaphysique de M. Enfantin est positivement celle de Hegel et que c'est à la suite de Hegel que l'école saint-simonienne s'est égarée " ${ }^{3}$. Qu'en est-il au juste des emprunts des saint-simoniens à la philosophie allemande dans sa globalité comme dans ses systèmes particuliers? Par quelles voies? Pour quels motifs? Selon quelle stratégie et quelle chronologie? Autant de questions auxquelles on peut espérer répondre à condition de considérer qu'il n'est pas un saint-simonisme, mais des courants saint-simoniens divers, qui ont des droits égaux à l'héritage de Saint-Simon.

1. Voir la traduction d'un riche compte rendu allemand de l'ouvrage de Veit qui est conservée dans le Fonds Enfantin de la Bibliothèque de l'Arsenal, Paris, Ms. 7825/11. 2. Voir Jacques D'HondT, «Hegel et les socialistes », La Pensée, 157, mai-juin 1971, p. 15 et 19 , n. 89 .

3. Revue indépendante, t. III, mai 1842, p. 332-333.

Revue de synthèse: IV S. No 2, avril-juin 1988. 
PHILOSOPHIE POSITIVE ET MÉTAPHYSIQUE ALLEMANDE

«Il [A. Mendelssohn] me fit connaître die Erziehung der Menschheit de Lessing, que je rapportai à Paris et communiquai plus tard aux saintsimoniens, ainsi que die Idee zu einer allgemeinen Geschichte de Kant. J'eus quelques conversations avec Hegel, auquel je remis la Politique positive de Comte. Il aimait beaucoup l'esprit pratique des Français. Il l'était fort peu lui-même [sic]."

G. d'Eichthal ${ }^{4}$.

Une contradiction du projet saint-simonien réside dans son ambition de transcender les sciences sans divaguer, de se proposer - pour user du syntagme paradoxal créé par Comte et adopté par le Producteur - comme une philosophie positive. C'est en ce point crucial qu'intervient l'Allemagne. Chez Saint-Simon, le débat des idées semble surtout lié à la rivalité des deux plus grandes puissances européennes, France et Angleterre. Mais, à y regarder de plus près, sa stratégie intellectuelle prend en compte un troisième partenaire. Au lendemain de la paix d'Amiens, il s'en serait allé "parcourir une partie de l'Allemagne", et en aurait rapporté « la certitude que la science générale était encore dans l'enfance dans ce pays, puisqu'elle y est encore fondée sur des principes mystiques "; mais il aurait aussi "conçu de l'espérance pour les progrès de la science générale [soit de la philosophie] en voyant la nation allemande tout entière passionnée dans cette orientation scientifique $»^{5}$. La ligne de partage passe désormais pour lui entre la «secte allemande" et la "secte anglo-française ». Vous avez raison, dit-il aux Allemands, de prétendre "que c'est seulement sous son rapport philosophique que la science est directement utile à la société ». N'exagérons pourtant pas la portée de cette tactique en supputant des connaissances approfondies : il n'en existe pas de trace. Aux yeux de Saint-Simon, la voie allemande est une impasse. Alors que, écrit-il en 1815, "l'Angleterre et la France se sont élevées [i.e. par la pensée] et ont élevé leurs gouvernements jusqu'à elles ", "l'Allemagne s'est élancée hors de son état social, et l'a laissé au-dessous d'elle " ${ }^{6}$. Or c'est déjà ce constat qui l'avait amené à prendre ses distances avec Villers,

\footnotetext{
4. Fonds Eichthal de l'Arsenal, Ms. $14408 / 10$.

5. Histoire de ma vie (1808), in Euvres, Paris, Anthropos, 1966, t. I, p. 70.

6. Travail sur la gravitation (1813), ibid., t. V, p. 299-300, et Réorganisation de la société européenne, ibid., t. I, p. 241.
} 
l'introducteur de Kant en France, et à critiquer son faible pour le luthéranisme ${ }^{7}$. Même attirance et même condescendance marquent le Nouveau Christianisme (1825).

C'est à partir de ces positions que Comte et Eichthal s'efforcent de gagner l'Allemagne à la «philosophie positive » et d'en détourner de l'eau pour leur moulin. La situation du premier à cette date est bien connue grâce aux travaux d'Henri Gouhier. Mais il faut tirer de l'ombre le second, alors seul et unique disciple de Comte, mais bientôt saintsimonien de marque, et premier lien du mouvement avec l'Allemagne ${ }^{8}$. Son rôle met en évidence la fonction de relais culturel exercée par le milieu juif franco-allemand auquel il appartient. C'est à son hôte à Berlin qu'Eichthal se dit redevable de son initiation : Abraham Mendelssohn, un ami de sa famille, père du compositeur et fils de Moses Mendelssohn, ce grand réformateur du judaïsme et intime de Lessing ${ }^{9}$. Et c'est à Comte qu'il confie la primeur de ses informations, dans une série de lettres échelonnées de mars 1824 à janvier $1825^{10}$. Le destinataire de ces rapports veut être renseigné «surtout sur les penseurs les plus rapprochés de [sa] tendance ». Averti par on ne sait quel canal de la division selon lui méconnue de l'École allemande « en École métaphysique et École historique », Comte estime que c'est la seconde fraction, où il nomme Herder, Buchholz, Heeren, Savigny et Meyer, qui pourra lui fournir « l'appui le plus fort, sinon le plus immédiat ». Aussi presse-til son correspondant de lui apporter confirmation de cette dualité ${ }^{11}$ si analogue à la discussion sans cesse renaissante en France des matérialistes et des spiritualistes. Telle est de fait la ligne adoptée par Eichthal au début de son exploration. Luden, préfacier des Idées sur une histoire philosophique du genre humain, ne note-t-il pas que chez Herder "l'Esprit n'est point présupposé, mais [...] semble se produire d'abord par l'organisation de la matière " ? Quant à Buchholz, " on peut dire qu'il a tout le système positif dans la tête; mais il n'y est pas seul [...] la métaphysique coexiste ${ }^{12}$. En fait, l'élève de Comte se tourne vite vers Kant et Hegel, quitte à convaincre son maître « que l'école historique dérive de Kant tout aussi bien que l'autre ». Quant à celle-ci - l'école philosophique -, son « vrai fondateur " ne serait

7. Introduction aux travaux scientifiques, ibid., t. VI, p. 205, n. 1.

8. Voir Barrie M. RATClifFE, "Saint-Simonism and Messianism : The Case of Gustave d'Eichthal », French Historical Studies, t. 9, 3, 1976, p. 484-502.

9. Voir supra n. 4.

10. Lettres publiées dans la Revue occidentale, 1896, p. 186-276, 345, 388. Larges extraits dans les Notes de la Correspondance générale et Confessions d'Auguste Comte, Paris, Mouton, 1973, t. I (cité par la suite comme Correspondance générale).

11. Correspondance générale, t. I, p. 80, 106-107.

12. Ibid., p. $386,383$. 
autre que... Rousseau : "C'est le seul de nos philosophes dont les Allemands tiennent encore compte, et c'est une chose très connue que c'est Rousseau qui a développé Kant. " De plus, le sage de Koenigsberg « est infiniment moins métaphysicien dans ses œuvres détachées que dans ses grands ouvrages ${ }^{13}$. Eichthal traduit donc l'Idée d'une histoire universelle du point de vue cosmopolitique. Et Comte convient que l'auteur est «le métaphysicien le plus rapproché de la philosophie positive ", ajoutant que si Condorcet, sa suprême référence, "avait eu connaissance de cet écrit, ce qu'il ne croilt] pas, il lui resterait bien peu de mérite ${ }^{14}$.

C'est à propos de Hegel que le décalage entre les deux hommes se fait le plus sensible. Eichthal travaille à persuader Comte de l'identité de sa philosophie de l'Histoire avec celle du penseur allemand dans les « résultats " sinon dans les principes, c'est-à-dire dans leur commune hostilité aux libéraux et aux apologistes du sentiment ${ }^{15}$. S'ensuivent quelques contacts personnels, plus diplomatiques qu'intellectuels, de juin à novembre 1824. Hegel lit l'essai de Comte, en apprécie la partie théorique, et reconnait aux "Français en général [...] un coup d'œil pénétrant dans le présent des choses, faculté tout à fait étrangère aux Allemands ». Mais il prouve derechef sa dernière assertion en déclarant "qu'il voyait peu à faire pour le côté pratique de l'entreprise; que dès qu'on passait à ce chapitre, tout devenait mesquin " ${ }^{16}$. Le philosophe positif concède à son tour à Hegel "un esprit positif dans les détails ", mais le situe bien au-dessous de Kant, et se refuse à confondre son Esprit si "singulier " avec le concept d' " esprit humain " tel qu'il l'a lui-même reçu de Condorcet via Saint-Simon ${ }^{17}$. Tout cela ne doit point faire illusion. Hégélien, Eichthal? Il a lu, sans « les comprendre totalement, faute d'être initié à la langue métaphysique ", des " esquisses de la Philosophie de l'Histoire " dispersée par Hegel dans ses ouvrages déjà publiés. Seul commentaire : Hegel a le «mérite d'avoir senti bien mieux que Herder l'enchaînement des phénomènes ". Il signale toutefois, à propos du "docteur Gans, qui se rendra bientôt à Paris ", qu'il «a écrit une histoire du droit d'héritage en établissant sa relation à chaque époque avec l'organisation sociale d'après les principes de la philosophie de Hegel ». Encore ne parait-il pas être allé au-delà de «l'introduction, qui est bien $"{ }^{18}$. Il est tentant de mettre cela en rapport avec la théorie

13. Ibid., p. 135, 143, 389.

14. Ibid., p. 143 .

15. Ibid., p. $391,393$.

16. Revue occidentale, 1896 , p. $228,257-258,271$.

17. Correspondance générale, t. 1 , p. 144.

18. Revue occidentale, 1896 , p. $228,260$. 
historique du droit de propriété développée dans l'Exposition de la doctrine saint-simonienne, et qui en est un point fort. Mais le bilan dressé le 12 janvier 1825 est désabusé : au total, Eichthal pense n'avoir recueilli qu'un «très petit nombre de données"; il n'a pas pris goût à « une activité philosophique [...] sans aucun rapport avec la vie pratique $"{ }^{19}$. C'est constater, comme Saint-Simon, que la philosophie allemande ne peut guère servir aux combats politiques français.

A plus long terme, dans des notes de 1863, Eichthal consigne la "grande sensation" produite dans le «monde saint-simonien" par sa traduction de l'opuscule de $\operatorname{Kant}^{20}$. Ce n'est pas un hasard si, en 1825, dans le Producteur, Comte est le premier, bientôt relayé par Enfantin, à user du concept d' " antagonisme " ${ }^{21}$. Il est vrai que l'origine allemande de l'idée est vite oubliée. A tel point que Buchez n'en découvre le contexte kantien qu'en 1828 , au plus tôt ${ }^{22}$. Il faut se reporter à l'Exposition pour observer le plein effet des rapports d'Eichthal. La seconde séance nomme officiellement Kant, Herder et Lessing parmi les ancêtres de la "doctrine ". La quatrième décrit le procès contradictoire et progressif de l'Histoire sous le concept d' "antagonisme " dans la perspective d'une "association universelle ". Mais de Hegel, point question. En revanche, l'admiration d'Eichthal pour l'Éducation du genre humain conduit les saint-simoniens à en publier la première traduction française, établie par Eugène Rodrigues, avec un large succès ${ }^{23}$. Précisons toutefois que rien n'autorise à prêter à cet apôtre, mort à vingt-deux ans, une quelconque familiarité de pensée avec Hegel : on sait seulement qu'il a traduit en 1824, pour Comte, des passages d'un ouvrage de Buchholz envoyé par Eichthal ${ }^{24}$. Quant à ce dernier, il paraît s'être complètement désintéressé par la suite de la philosophie allemande.

19. Correspondance générale, p. 396.

20. Fonds Eichthal de l'Arsenal, Ms. 14 406/4.

21. Voir éd. Bougle de l'Exposition, n. 93.

22. Introduction à la Science de l'Histoire, p. 94-98. Buchez suppose que Saint-Simon a lu la traduction parue dans le Conservateur en 1801, mais "antagonisme " n'apparaît pas sous sa plume.

23. Il $\mathrm{y}$ eut trois éditions.

24. Correspondance générale, t. I, p. 96. 


\section{L'AFFLUX DES DEÇUS DE L'ECLECTISME}

"Allons un peu à cette Allemagne qui prêta sans le savoir l'autorité de son nom à cette déception métaphysique. "

E. Lerminier, $1833^{25}$, De l'influence de la philosophie du xviiï siècle sur la législation et la sociabilité du xixe siècle

Fin 1828 début 1829, quelques-uns des plus brillants fervents de Victor Cousin et de Guizot, reprennent ce flambeau : Jules Lechevalier, Eugène Lerminier, Henri Lagarmitte. Comme leur ardeur ne pouvait se satisfaire de la modération de leurs aînés, ils avaient cherché chez les maîtres allemands de leurs maitres français une vigueur originelle, avant que de reconnaitre dans le saint-simonisme la synthèse radicale propre à combler leurs aspirations.

Le premier ${ }^{26}$ a voyagé deux ans durant en Allemagne, vers 1827-1828, avec le projet particulier de "s'assimiler Hegel " ${ }^{27}$. A son retour, il prend place dans le petit cercle des proches d'Enfantin. Ce sont eux qui attestent et dénoncent cette prédilection. Ils la considèrent, en effet, non pas comme une voie de passage vers leur doctrine, mais bel et bien comme un obstacle idéologique majeur. Enfantin triomphe en avril 1830 :

"La métaphysique de Lechevalier est décidément enfoncée, nous avons l'autre jour, Bazard et moi, retourné le portrait d'Hegel qui était dans sa chambre, et écrit sur le dos :

SAINT-SIMON

$$
\text { SCIENCE RELIGION INDUSTRIE }{ }^{28} \text {. }
$$

Mais deux mois plus tard, Eichthal, dont on peut ici mesurer l'évolution, déplore encore l'inaccomplissement de la rupture. Lechevalier, moucharde-t-il auprès d'Enfantin, se serait fourvoyé dans une discussion "métaphysique " digne de Hegel et des "sectes philosophiques ", mais non d'un apôtre brûlant de foi. L'hégélianisme, insiste-t-il,

25. Cf., p. 309 (à propos de l'éclectisme).

26. Voir Notice sur Jules Lechevalier et Abel Transon, Paris, 1877, par Charles Pellarin, et Dictionnaire biographique du mouvement ouvrier, Paris, 1964, de Jean MAITRON.

27. Fonds Enfantin, Ms. $7804 / 2$.

28. Correspondance d'Enfantin, in Euvres d'Enfantin, Paris, 1872, t. VII, p. 102. 
ressortit à la "critique ${ }^{29}$. Autrement dit, il est à la fois trop métaphysique - grief repris de Comte -, et trop rationnel, trop philosophique, pas assez religieux - grief inédit, déduit de la mutation fidéiste du saint-simonisme. A lire le brillant compte rendu que Lechevalier consacre, en 1833, dans l'Europe littéraire, aux Mélanges de Jouffroy, il apparait cependant qu'il n'a pas pu ne pas contribuer à démarquer «la doctrine" des "doctrinaires ». Il accorde certes le label germanique à Cousin, dont il détaille les emprunts à Berlin. Mais c'est pour mieux rappeler le jugement motivé de Hegel sur l'éclectisme : "philosophie tout à fait vide (ganz leere)." A l'inverse, Lechevalier souligne le caractère "progressif » de la dialectique hégélienne, dont il donne une analyse parfaitement informée : "Le système qui représente la catégorie inférieure est conservé, absorbé (aufgehoben), assimilé et reproduit par le système supérieur, jusqu'à ce que l'esprit humain arrive à s'identifier avec la vérité absolue $"{ }^{30}$. Pour mesurer l'apport de Lechevalier, il n'est qu'à lire sa leçon publique du 20 janvier 1831. Titre : "Prolégomènes généraux. " Sujet : la philosophie de l'Histoire. Thèse : "L'humanité, comme tout être vivant, a sa loi : Vico, Montesquieu, Kant, Lessing, Herder, Condorcet, Turgot, Hegel, l'ont cherchée, SAINT-SIMON l'a trouvée. " Et de développer l'idée que « deux principes ", la matière et l'esprit, constituent et dirigent la vie, que ces principes ont toujours exercé leur hégémonie en opposition l'un à l'autre, et alternativement, mais que le saint-simonisme va enfin les faire accéder à un état d'harmonie définitif ${ }^{31}$. Or ce schéma ternaire et ascensionnel constitue la trame de la philosophie de l'Histoire propagée par les saintsimoniens jusqu'à leur renoncement à des perspectives révolutionnaires, soit jusqu'au schisme de Bazard.

Un autre mérite de Lechevalier est d'avoir «beaucoup contribué à la venue de Lherminier [sic] par le lien commun de l'Allemagne ${ }^{32}$. Mi-strasbourgeois, mi-parisien, Eugène Lerminier avait rapporté d'un séjour auprès de Savigny une thèse sur le droit de propriété. Les relations de cet universitaire avec le mouvement saint-simonien sont méconnues. Il est pourtant le premier des républicains du Globe de Dubois à franchir le pas, en 1830, avant même Leroux ${ }^{33}$. Sa fuite précipitée le 13 novembre ne rompt pas les liens idéologiques. Car Lerminier ne se pose guère qu'en s'opposant à ses anciens amis - par exemple sur la question de la Propriété - et ses idées les plus audacieuses

29. Fonds Alfred Péreire, Bibliothèque nationale, Mss, N.A.fr. $24609, \mathrm{f}^{\circ} 479$.

30. Art. cit., oct.-nov. 1833, p. $125,127$.

31. Organisateur, $2^{\mathrm{e}}$ année, 24, 1830, p. 189.

32. Fonds Enfantin, Ms. $7804 / 2$ (notice sur " Jules").

33. Ibid. (notice sur Leroux). 
sont aussi les leurs: l'émancipation des femmes, la nécessité d'un ressourcement en Orient, la prophétie d' « un révélateur intelligent et intelligible $"{ }^{34}$. Son Introduction générale à l'histoire du Droit (1829) traite en réalité de la philosophie de cette discipline, et s'appuie surtout sur les auteurs allemands. Y sont analysés les écrits et le rôle de Savigny, mais aussi ceux de Hegel et de Gans, et, avec plus d'insistance, la tradition directement issue de Kant. Lerminier néglige cependant le thème cosmopolite et pacifiste, et préfère souligner, à la suite de Cousin, que le criticisme interdit radicalement toute ontologie et toute morale. Le patronage éclectique est toutefois jeté aux orties en même temps que le costume saint-simonien dans la Philosophie du droit (1831). Lerminier s'y félicite d'avoir « le premier en France prononcé le nom et fait connaître quelques idées de Hegel, mais [précision perfide visant Cousin] en lui en renvoyant la gloire ». Le jeune professeur n'en attaque pas moins rudement le maître de Berlin : son système ne serait « qu'une logique hérissée de formules", sa dialectique serait "sans bornes et sans rivages ", sa proposition sur l'identité du réel et du rationnel nierait toute liberté. Le premier exposé en France du «panthéisme scolastique » en est un véritable éreintement. Selon Lerminier, Hegel méconnaît la force émancipatrice du spiritualisme chrétien, prêche la résignation devant « le pouvoir", "le despotisme ", "les maux de l'humanité ", et, pour finir, « blâme jusqu'aux efforts que fait un peuple dans le cercle de la loi pour réformer sa constitution " ${ }^{35}$. C'est anticiper, au négatif, sur le discours néo-catholique et révolutionnaire des buchéziens (voir infra). Trouvent grâce en revanche Lessing, qui a su résumer « ce qui a été dit dans le XvIIr siècle de plus profond et de plus net sur la religion et le christianisme "; Kant, qui a montré la religion « d'accord avec la raison » en morale, et s'est intéressé à la Révolution française ; le "généreux Fichte", auteur du Droit naturel, qui, ayant atteint la pointe extrême de l'idéalisme - l'homme sans Dieu et sans le Monde -, est revenu au « réalisme »; Schelling, enfin, qui pèche par abstraction, mais qui, d'accord avec Goethe, a su montrer dans la poésie et le symbole, les voies de la révélation divine ${ }^{36}$.

Une révision du procès de Hegel s'opère cependant dans $\mathbf{A u}$-delà $d u$ Rhin (1835). Le "logicien du panthéisme moderne" (on note le changement d'épithète) est toujours accusé de n'avoir pas « senti » que « l'idée du droit est mobile », mais son œuvre apparaît non plus comme

34. Au-delà du Rhin, Paris, 1835, t. II, p. 271-272; "De la propriété ", Revue des Deux Mondes, $2^{e}$ sem. 1831, repris dans Lettres philosophiques adressées à un Berlinois, Paris, 1832, p. 477.

35. Op. cit., t. I, p. XLVII, 199, 201, 204, 214, 216.

36. Ibid., t. II, chap. VII et $\mathrm{Ex}$, passim. 
une impasse, mais comme la synthèse de toute la philosophie passée, et le point de départ possible de "développements nouveaux et féconds $"{ }^{37}$. Ce revirement ne s'explique pas que par la propagande de Gans, le destinataire putatif des Lettres philosophiques adressées à un Berlinois (1832). Il s'inscrit dans une perspective mystique, l'hypothèse cyclique que, "comme l'idéalisme grec a préparé le christianisme, l'idéalisme germanique prépare la religion qui succédera au christianisme ${ }^{38}$ : d'où l'importance accordée à Novalis et à Baader. Trois ans plus tôt, comme pour mettre en garde son Berlinois contre la tentation de reconnaitre le saint-simonisme dans cet après-christianisme, il insistait sur sa conviction que "depuis Jésus-Christ, le panthéisme n'est plus socialement possible ", et il accablait sa foi passée sous les reproches de "contemplation oisive", de mépris matérialiste de la liberté et de l'individualité ${ }^{39}$. Mais qu'avait-il au juste dans sa ligne de mire? le saint-simonisme, ou la philosophie allemande? ou encore l'intérêt de certains hégéliens pour le saint-simonisme? Sans doute les trois objectifs à la fois, visés en enfilade ou par ricochets. La notoriété de Lerminier, ses liens avec Leroux, le rayonnement de la Revue des Deux Mondes, dont il est un élément en vue, donnent en tout cas à ses positions un poids certain dans l'intelligentsia saint-simonienne et saint-simonisante des années 1830 .

Henri Lagarmitte ${ }^{40}$ est lui aussi un représentant de l'interculturalité strasbourgeoise. Initié à la jurisprudence allemande par son parent Mittermaier (un tenant de l'école historique, professeur à Heidelberg), déçu par la politique de ses idoles doctrinaires, mais enthousiasmé par l'Exposition, il monte à Paris en juin 1831 et prend en charge les nouvelles de l'Allemagne dans le Globe, à partir, au moins, de janvier 1832, jusqu'à la fin du journal, à la mi-avril. Pressenti pour appartenir au " corps apostolique " de Ménilmontant, il se dérobe, puis écrit dans le Temps et dans la Revue encyclopédique de Leroux, toujours sur l'Allemagne. Par ses origines, sa formation et ses projets, Lagarmitte fait figure d'un second Lerminier. La vision de la philosophie allemande qu'il exprime dans le Globe ne se signale ni par l'ampleur ni par l'originalité. De Kant, il mentionne dans une note, sans plus, l'opuscule pour une " histoire universelle ". En une phase où le saint-simonisme, sous l'impulsion d'Enfantin, prône une politique de " conciliation " non sans analogie avec le juste milieu, Lagarmitte, jouant les éclaireurs de

37. Op. cit., p. 105, 138, 139, 137-138.

38. Au-delà du Rhin, op. cit. supra n. 34 , t. II, p. 147.

39. Revue des Deux Mondes, t. VII, 1832, p. 476-477, 481.

40. Voir Marcel EMERrT, "Les saint-simoniens à Strasbourg ", Annales de l'Est, série 5, 1,1976 , p. $65-78$, et Revue germanique, juin 1834, p. 170 sqq. 
ce rapprochement, trouve sans doute plus actuel d'évoquer «l'illustre Hegel " comme "l'inspirateur des principales conceptions de l'école éclectique ". Tout en faisant grief aux doctrinaires d'avoir voulu arrêter le mouvement de l'Histoire à la Charte de 1814 , et de s'être ainsi malencontreusement liés au sort de la Restauration, il leur tresse, en effet, des couronnes pour avoir eu la sagesse, par rapport aux purs libéraux, de redescendre "des hauteurs nébuleuses de l'utopie sur le terrain fécond de la réalité ", suivant en cela le célèbre précepte de l'Introduction au Naturrecht, "seine Zeit im Gedanken zu erfassen". L'école de Savigny lui paraît d'ailleurs prôner le même pragmatisme, ce que prouverait l'égal intérêt du gouvernement prussien pour les deux factions rivales ${ }^{41}$. Un doute toutefois tenaille Lagarmitte: Hegel n'aurait-il pas, "par sa fameuse devise: "tout ce qui existe est raisonnable" [sic], paralysé d'une manière mortelle le sentiment du progrès, le dévouement qui inspire les hommes à se sacrifier pour une noble cause" ? n'aurait-il pas "fait fi de la liberté, de l'individualité $"{ }^{42}$ ? Le propos est évidemment emprunté à la topique de Lerminier. Aussi bien Lagarmitte, dans une lettre à Enfantin du 6 octobre 1831, s'ouvrait-il déjà de la perplexité où l'avait plongé l'enseignement de ce jeune aîné, quant au socialisme saint-simonien - le néologisme ne va pas tarder à apparaitre, en mauvaise part, dans le Globe $e^{43}$. L'impressionne, en effet, cette " observation ", selon lui empruntée à l'Allemagne,

«que, depuis l'origine de l'humanité, deux principes se sont disputé l'organisation sociale, le principe de la sociabilité et celui de l'individualité, qui, sous d'autres manifestations, ont produit le dualisme qu'on a remarqué entre la liberté et la fatalité, entre la volonté et l'intelligence, entre le monde subjectif et objectif $\left[\right.$ sic ${ }^{*}{ }^{44}$.

Il n'est pas difficile de reconnaître dans cette problématique, outre Fichte vulgarisé par Cousin, le Kant de l'opuscule sur «l'Histoire universelle " susmentionné, et sa formule de l" "unsittliche Sittlichkeit" (l'insociable sociabilité). Tant il est vrai qu'en matière de logique, Lagarmitte en est encore aux antinomies kantiennes, comme beaucoup d'autres.

41. Glabe, 29 janvier et 7 avril 1832 .

42. Ibid., 7 avril 1832 .

43. Ibid., 13 février 1832 .

44. Fonds Alfred Péreire, Bibliothèque nationale, Mss, N.A.fr. 24610 , ff. 57-59. 


\section{IDENTITÉ NATIONALE ET PHILOSOPHIE DE L'IDENTTTÉ ABSOLUE}

« Le temps approche où il n'y aura plus une ou plusieurs philosophies allemandes, une ou plusieurs philosophies françaises, mais où il n'y aura plus qu'une philosophie, qui sera en même temps une religion."

Pierre Leroux, 1842. «C'est à l'avenir de décider quelle tendance prévaudra, de la tendance allemande ou de la tendance française. Ce sera l'une ou l'autre, mais certainement pas toutes les deux."

Auguste Ott, $1844^{45}$.

Le Globe, pris en main par Michel Chevalier à partir du 11 novembre 1830, observe un silence pesant jusqu'en mars 1831, date à compter de laquelle Goethe, Lessing, Kant et Hegel sont fréquemment mentionnés parmi les prophètes d'une nouvelle ère religieuse. Il est tentant de rapporter cette espèce de censure à la ligne radicale suivie par Bazard et Enfantin jusqu'au début de l'hiver de 1831. Mais il faut aussi prendre en compte le départ de Lerminier. En septembre et en octobre 1830, en effet, le compagnon de Leroux, pressé par les saint-simoniens de " [s']associer à leurs efforts pour travailler [lui]-même au but qu'[il] se proposai $[t]{ }^{46}$, denne plusieurs articles dans lesquels il semble adopter pour lui-même et ses amis le projet de son maître Cousin d'intégrer la philosophie allemande à une philosophie nouvelle et française. Se posant en médiateur entre "les successeurs de Kant" et les adeptes de l'école historique, il propose de les prendre indistinctement " pour notre point de départ ", à nous Français, afin de "marcher à quelque chose d'indigène et de national $"{ }^{47}$. Cette tâche nationale, en vérité, sert ses ambitions, à l'intérieur, puis à l'extérieur du groupe saintsimonien. La germanophilie de Lerminier plagie le plagiat si haut dénoncé de Cousin tout en s'en distinguant. Elle s'accompagne donc d'un patriotisme agressif, d'une exaltation compensatoire du XvIII siècle, c'est-à-dire de la philosophie spécifiquement française. C'est ainsi qu'en 1833, devenu le chef de file des républicains de l'ancien Globe réfugiés à la Revue des Deux Mondes, il établit un parallèle inattendu entre la Critique de la Raison pure et le Traité des sensations de Condillac, expression, selon lui, d' "un idéalisme sensitif». Il compare aussi

45. Conclusion de l'article sur le "Cours de philosophie de Schelling ", Revue indépendante, mai 1842, p. 348 ; A. OTT, Hegel et la philosophie allemande, Paris, 1844, p. VII.

46. Philosophie du Droit, Paris, 1831, p. XXV.

47. Article sur l'Histoire du Droit romain de SAVigNY, 6 sept. 1830 (même idée le 14 oct. à propos de Ballanche). 
avantageusement le panthéisme populaire de Diderot au panthéisme aristocratique de $\mathrm{Hegel}^{48}$.

L'effacement de toute polémique nationale, sinon de tout complexe de supériorité, caractérise au contraire la ligne du Globe enfantinien, en conformité avec la ligne pacifique promue par le Père suprême et traduite le 3 juin 1831 par Chevalier. Ce dernier reprend à son compte la division internationale du travail naguère proposée par Cousin entre France, Angleterre et Allemagne (associer les peuples, les initier à l'industrie, leur distribuer la science), et il avoue sa préférence pour la Prusse " au sein de l'Allemagne ». Du coup, Lagarmitte se démarque des germanistes jacobins de la Revue des Deux Mondes, notamment d'Edgar Quinet et de sa violente dénonciation de l'impérialisme prussien. Il incite, lui, les saint-simoniens à réactiver la pratique des échanges intellectuels franco-allemands en vigueur dans le Globe libéral - un « héritage " qui, "depuis dix-huit mois", " menaçait] de tomber en déshérence ". Car ils ne risquent pas de se laisser aller comme leurs prédécesseurs à des «idées de fusion, d'abdication de l'individualité nationale ", et d'encourir, comme eux, le "reproche [...] de prétendre imposer à la France la science allemande, comme la France, sous Napoléon, aurait imposé à l'Allemagne ses codes et ses administrateurs " : ils sont les «disciples d'une doctrine sortie des entrailles de l'individualité française, puisqu'elle est le dernier point de la ligne droite qui a été parcourue par Descartes, Voltaire, Condorcet». C'est en possession de ce précieux dépôt qu'ils se présentent face « aux hommes de la ligne droite allemande, de cette ligne dans laquelle ont marché Luther, Leibniz, Kant et Hegel ". Or ces deux lignes en sont, selon Lagarmitte, à un tel "point de rapprochement " qu'il est désormais possible d'œuvrer à "la grande association" prophétisée par SaintSimon $^{49}$. Aussi Lagarmitte s'emploie-t-il, à l'intention de ses lecteurs allemands, à contrebattre la réputation d'utopie révolutionnaire acquise par le saint-simonisme, en même temps qu'il fait miroiter à ses lecteurs français le caractère organique et religieux de la philosophie de Hegel, et s'efforce d'en compenser l'image fataliste et autoritaire, prussienne, pour dire le mot, par le tableau de l'agitation libérale en Rhénanie ${ }^{50}$. Ces analyses ne sont cependant guère suivies. Son expédition d'Égypte marque, en effet, pour Enfantin, un retour à des préoccupations essentiellement industrialistes. Ni le contenu de l'essai de Heine sur

48. De l'influence de la philosophie du xvII siecle sur la législation et la sociabilité $d u$ XIX siecle, Paris, 1833, p. 97-99, 72-73.

49. Globe, 16 janvier 1832 .

50. Ibid., 7 avril 1832. 
l'Allemagne, ni la flatteuse et fameuse dédicace qui lui en est faite, ne le convainquent de relancer l'alliance intellectuelle franco-allemande. Bien au contraire. Dans sa réponse, il déclare que " tous les grands noms philosophiques de l'Allemagne" sont désormais à ses yeux "du passé [...], une histoire». Il exprime, en conséquence, le vœu que Heine abandonne le terrain philosophique pour étudier « la situation politique, morale, artistique et industrielle de l'Allemagne ", et travailler à rapprocher les jeunes générations des deux pays. Enfantin, en d'autres termes, recommande, contrairement à Heine, que l'on cesse de "montrer [...] la communion de doctrines du nord de l'Allemagne avec la France", et de «faire sentir [...] la communauté d'intérêts des États du Rhin et de la France ". Et il préconise à l'inverse de se tourner vers... l'Autriche, en tant qu'elle représente l' " âme " et la tradition germaniques ${ }^{51}$.

Lechevalier lui-même pousse la mauvaise foi, en 1832, jusqu'à prétendre n'avoir jamais nourri d'illusions sur la philosophie allemande. Dans le Phalanstère, il déclare s'être rendu en Allemagne "pour vérifier", " malgré la trop grande importance attachée par l'éclectisme aux travaux des Allemands", "que le véritable mouvement des idées était en France et s'opérerait en continuant, à un point de vue plus élevé et en ordre inverse, les travaux du XVIII siècle". S'il y a bien outre-Rhin "d'immenses matériaux accumulés ", le " principe " d'organisation manque absolument, et la Révolution française a creusé un écart décisif ${ }^{52}$. Au surplus, Lechevalier suggère a contrario l'allogénéité et la nature éclectique du saint-simonisme en se vantant d'avoir «le premier [...] parmi les saint-simoniens " perçu le « caractère autochtone, homogène, sans alliage " de la doctrine de Fourier, comparée à « l'espèce de doctrine d'alluvion ", à " la vraie tentative de syncrétisme de notre siècle " qu'est, selon lui, la doctrine "agglutinée et agglomérée sous le nom de Saint-Simon " ${ }^{53}$.

Tout aussi ambivalente est l'attitude de Pierre Leroux, à partir des années 1840 , dans une conjoncture marquée par l'activité des Jeunes Hégéliens et les coquetteries germaniques de Proudhon. Il prend position, en vérité, bien plus qu'il ne témoigne, lorsque, explicitant les allusions de Lechevalier, il réédite contre Enfantin la manœuvre de Lerminier contre Cousin : accusation de plagiat de Hegel, imputation d'une inflexion conservatrice à l'influence berlinoise, puis récupération du prestige germanique. Premier temps de la dernière opération : Leroux

51. Heine, De l'Allemagne, Paris, Charles Duguet, s.d., p. 5, 6-7, 11-13.

52. Phalanstère, 26 juillet 1832.

53. Art. cit. supra n. 30 , ibid. 
applique à Hegel le topos créé par les saint-simoniens pour le $\mathrm{XVIII}^{\mathrm{e}}$ siècle, estimant que, "comme la philosophie de Voltaire", sa métaphysique est une " critique ", contenant donc, sous forme négative, « une portion de vérité ». Second temps : il assure que la "moralité " de son cuvre serait réductible à « l'esprit qui anima Condorcet et SaintSimon". Mieux, "la philosophie de Schelling et de Hegel» (sic) ne serait rien de plus que «l'analyse de la philosophie française et contemporaine" - entendons ce que Leroux lui-même extrapole du $\mathrm{XVIII}^{e}$ siècle et de naturalistes comme Geoffroy Saint-Hilaire ${ }^{54}$. Pour le philosophe socialiste, ces manipulations herméneutiques sont un puissant motif de confiance dans la validité de son système comme dans la fécondité d'une rencontre intellectuelle entre France et Allemagne, la base de la "Nouvelle Alliance de l'Humanité ". Cette inspiration doit beaucoup au récent retour de Schelling, remonté sur la scène philosophique pour réfuter Hegel, et, du même coup, croit comprendre Leroux, ce panthéisme en quoi il voit la clé de voûte du dogme enfantinien de la « loi vivante », c'est-à-dire de la concentration de tous les pouvoirs entre les mains d'un Père suprême, à l'image de l'incarnation de l'infini dans le fini. Pour Leroux, en somme, "Schelling croit à un nouveau christianisme " ${ }^{55}$. Le mot est lâché : le patriarche de la philosophie allemande est un saint-simonien, ou un leroussien, qui s'ignore. Ce "syncrétisme " de Leroux, avoué comme tel, ressemble fort au saint-simonisme tel que le rêvait Lerminier en 1830 , tel que le décrivait Lechevalier en 1831, et tel enfin, à part l'ingrédient fouriériste, que l'analyse, en 1846, Leroux lui-même, mimant la dialectique germanique : un "synchrétisme " (sic) réunissant « les idées saines de Saint-Simon et les idées malsaines de Fourier, au moyen d'un troisième terme, la métaphysique à moitié vraie, à moitié fausse de Hegel ${ }^{56}$.

Une seule exception à cette germanophilie équivoque, mais si prégnante : les buchéziens, pour qui l'individualisme protestant ne saurait se concilier avec le "point de vue social ", car catholique, des Français. Mais il est un préjugé sur lequel Ott, porte-parole du groupe en ce domaine, s'accorde implicitement tant avec Lerminier qu'avec Leroux contre Enfantin : le "panthéisme " débouche sur un fatalisme conservateur ${ }^{57}$.

Tout se passe, en fin de compte, comme si les saint-simoniens ne

54. Revue indépendante, t. III, 1842, p. 324-325 (rééd. 1982, coll. "Vrin Reprises ").

55. Ibid., p. 21 et 334.

56. Revue sociale, 1846 , p. 145.

57. A. OTT, op. cit. supra n. 45, p. VII et passim (cf. article du même contre Proudhon germanisant, Revue nationale, juin 1847). 
pouvaient s'intéresser à la philosophie allemande qu'en fonction de ses possibles effets et utilisations dans le contexte français : les conditions et les objectifs de leurs importations théoriques déterminent leur sens, c'est-à-dire le limitent et l'orientent à la fois. On perçoit par là combien il serait erroné d'opposer à une imprégnation germanique profonde et quelque peu mystérieuse, les manœuvres, les figures de pensée, auxquelles se livrent les saints-simoniens - en surface, serait-on tenté de dire - pour élaborer une représentation de la philosophie allemande à leur convenance, et, forts de cette spécialité dans le Savoir, conquérir un pouvoir intellectuel.

L'Allemagne néanmoins semble proposer une synthèse heureuse des antinomies qui déchirent leur identité nationale. Mais, comme le notait Saint-Simon, son arriération politique contraste avec son surdéveloppement philosophique et le met en question. Image inverse de la France, elle ne peut lui servir de modèle, mais seulement de miroir.

Ainsi de Hegel. Le ressort "progressif " de sa dialectique décelé par Lechevalier ne contrebalance pas sa réputation d'éclectique ni son prétendu panthéisme, qui séduisent les dirigeants les plus antichrétiens (notamment Enfantin), en même temps les moins ardents à la subversion. Le topos dominant, l'opposition amalgamante panthéisme-matérialismefatalisme-conservation sociale $v s$ spiritualisme-liberté-individualismeprogrès social ${ }^{58}$, fonctionne ici à plein régime. Ceux-là mêmes qui se réclament du XviI ${ }^{\mathrm{e}}$ siècle, de la Révolution et de la République (Buchez, Leroux, Lerminier à ses débuts...) se trouvent contradictoirement déportés vers les valeurs néocatholiques, et, du même coup, durablement, sinon définitivement écartés de la philosophie allemande post-kantienne. Il faut toute l'équivoque du Retour de Schelling pour que Leroux, une décennie plus tard, se décide à prendre langue avec l'Allemagne. Mais le dialogue n'est-il pas alors faussé par le précédent des années 1830 ? Philippe RÉGNIER,
C.N.R.S.

58. Voir Images au XIX siècle du matérialisme du XVIIt siècle, dir. Olivier BLOCH, Paris, Desclée, 1979. 